\title{
Mathematical Tools for Analysis, Simulation and Design of Robotic Angular Encoders
}

\author{
Peter G. Cramer [16]
}

\begin{abstract}
A set of practical mathematical tools for creating self correcting, self compensating optical angular encoders is presented. Included is [a] a discussion and proof of the so-called Angular Encoder Theorem, which is the fundamental element of real time self correction based on read head symmetry averaging; $[b]$ a discussion and derivation of the self-compensation equations, which can be used in tandem with the encoder theorem to increase accuracy further; [c] a kinematic model which can be used to simulate realistic operational scenarios; and [d] rules of thumb and implications for the designer. Sample simulation results and sample test results are also presented. These tools enable the designer to work out cost-effective encoder designs to meet different accuracy requirements for different applications. Such encoders automatically retain their accuracy over indefinite periods of time without human intervention of any kind and are thus well suited for challenging robotic applications. These tools can and have been used to construct cost-effective encoders with demonstrated sub-arcsecond accuracy. Finally, sample source code is available online.
\end{abstract}

\section{INTRODUCTION}

An essential requirement for more accurate robots is more accurate angular encoders. In recent years, much progress has been made. New high precision calibration methods have been developed by Estler et al.[1], Masuda et al.[5], [7], Lu et al.[9] and Just et al.[8]; new self-compensation techniques have been developed by Geckeler et al.[2], Probst [3], Watanabe et al. [6], Masuda[5] and Cramer [10]; and self-correction by read head symmetry averaging has been investigated experimentally by Watanabe et al.[4] and Cramer [10]. The purpose here is to put these results onto a common mathematical footing, enabling designers to create practical encoders for many different robotic applications.

(For clarity the following terminology, taken from the metrology field, is used here: compensation is the measurement of parameters which govern the systematic errors of the device, correction is the adjustment of raw measurements to improve accuracy, and calibration is the comparison of the device under test with a trusted reference that is directly traceable to a national standards agency.)

Most promising is the optical angular encoder, which has the great advantage of being non-contact and therefore free from errors caused by direct physical contact, free of friction and mechanical wear, and free from stick-slip behavior for optimum control system performance.

A typical spindle assembly is shown in figure 1 Two bearings hold a rotating shaft in place while allowing it to rotate about its longitudinal axis. The outer races of the bearings are fixed to the stator which is not shown. The angular encoder assembly is on the right side of the spindle. The

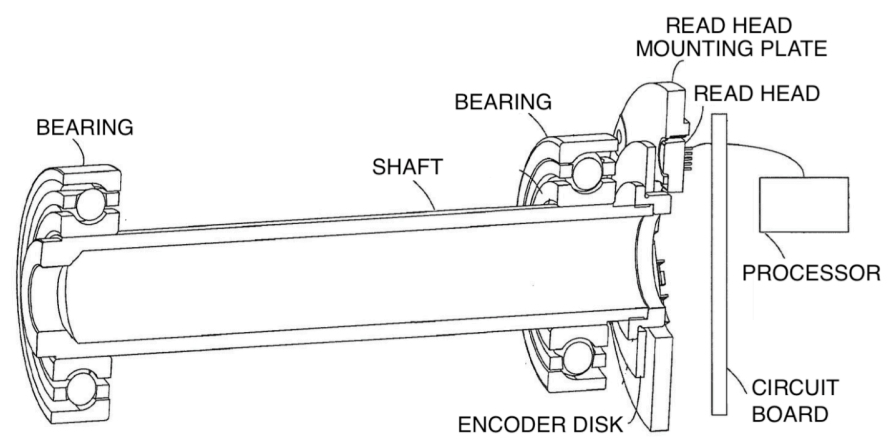

Fig. 1. Spindle assembly with angular encoder

rotating part is the encoder disk which is fixed to the rotating shaft. The non-rotating part consists of one or more read heads which are rigidly attached to a mounting plate. The read heads are soldered to a circuit board which in turn is connected to an on-board processor.

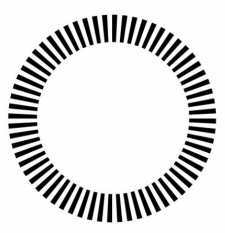

Fig. 2. Encoder disk diffraction grating (number of lines greatly reduced for clarity)

The rotating encoder disk, typically made of glass, has a series of radial lines etched on its surface, as shown schematically in figure 2. These lines form a diffraction grating, and the read head design takes advantage of the diffraction effect to achieve a high level of precision. A typical encoder disk diffraction grating has on the order of 10,000 lines, and an angular resolution of well under $1 \mu \mathrm{rad}$ is readily achievable with standard commercial components.

The layout of a typical optical read head is shown in figure 3. An LED in the head emits a coherent beam of light which strikes the diffraction grating etched on the encoder disk. (At high magnification the grating has the appearance of a straight line, but actually the grating is circular and the individual lines radiate from a common center which ideally lies on the axis of rotation.) The beam is reflected by the disk grating, passes through an index grating and then falls on a set of photodiodes. Diffraction effects are used to obtain high precision quadrature measurements of the position of the read head relative to the 


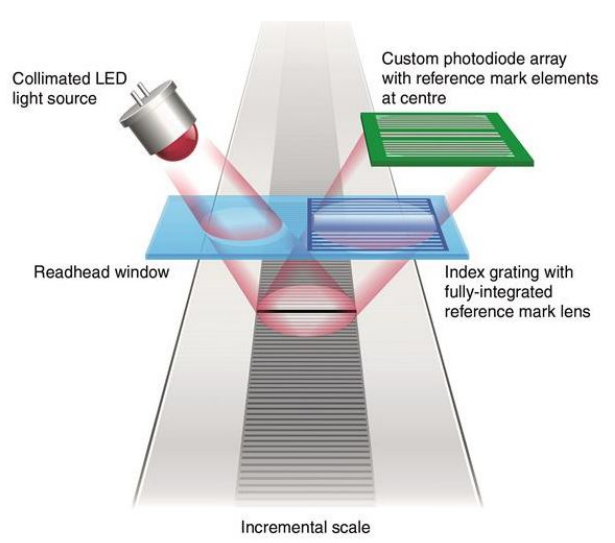

Fig. 3. Optical read head concept (courtesy renishaw.com)

two nearest grating lines, i.e. the two lines which bracket the read head.

\section{The Angular ENCODER TheOrem}

Perhaps the single most important tool for the encoder designer is the angular encoder theorem. It provides a simple and cost-effective way to increase encoder accuracy without imposing overly strict mechanical tolerances.

\section{A. Theorem Statement}

Consider an angular encoder with $N$ equally spaced read heads, and define the read head average as $\bar{\theta}=\Sigma \theta_{i} / N$, where $\theta_{i}$ is the angle measurement of read head $i$. Assume that the error is continuous and periodic with period $2 \pi$. Assume also that the error is a function the disk rotation angle only, and that each read head is subject to the same error modulo a phase angle given by its location relative to the other read heads. Then all Fourier error modes cancel identically except for mode $N$ and its harmonics. In other words, only error modes $N, 2 N, 3 N,$. . remain after averaging. For clarity this will be called symmetry averaging to distinguish it from ordinary statistical averaging, which at best provides only $\frac{1}{\sqrt{N}}$ behavior.

\section{B. Proof}

Consider an encoder with $N$ equally spaced read heads. The read head spacing is $2 \pi / N$, and the read head average is

$$
\bar{e}(\theta)=\frac{1}{N} \sum_{h=1}^{N} e_{h}(\theta)=\frac{1}{N} \sum_{h=1}^{N} e(\theta+2 \pi h / N) .
$$

where $h$ is the head number. The error due to mode $m$ is

$$
\begin{aligned}
\bar{e}_{m}(\theta) & =\frac{1}{N} \sum_{h=1}^{N} e_{h m}(\theta) \\
& =\frac{1}{N} \sum_{h=1}^{N} e_{m}(\theta+2 \pi h / N) \\
& =\frac{1}{N} \sum_{h=1}^{N} \sin (m \theta+2 \pi m h / N+\phi) .
\end{aligned}
$$

Now consider the case in which $m$ is a harmonic of $N$, i.e., $m=j N$ where $j=1,2,3, \ldots$. The mode $m$ error is

$$
\begin{aligned}
\bar{e}_{m}(\theta) & =\frac{1}{N} \sum_{h=1}^{N} \sin (m \theta+2 \pi j N h / N+\phi) \\
& =\frac{1}{N} \sum_{h=1}^{N} \sin (m \theta+2 \pi j h+\phi) \\
& =\frac{1}{N} \sum_{h=1}^{N} \sin (m \theta+\phi)=e_{m}
\end{aligned}
$$

This proves that error modes which are harmonics of $N$, the number of read heads, are unaffected by symmetry averaging.

Next consider the case of error modes which are not harmonics of $N$. In this case one can write $m=j N+k$, where $j$ is a positive integer and $k=m \bmod N$. The argument of the sine function can be written as

$$
\begin{aligned}
m \theta+2 \pi m h / N+\phi & =m \theta+2 \pi(j N+k) h / N+\phi \\
& =m \theta+2 \pi k h / N+\phi .
\end{aligned}
$$

This is a useful simplification, as $1<k<N-1$. If the theorem holds for these modes, then it holds for higher modes as well. Now it is only necessary to prove that

$$
\bar{e}_{m}(\theta)=\frac{1}{N} \sum_{h=1}^{N} \sin (m \theta+2 \pi m h / N+\phi)=0
$$

for $1 \leq m<N$. Using a standard trigonometric identity, the sine term can be written in the form

$$
\sin (m \theta+\phi) \cos (2 \pi m h / N)+\cos (m \theta+\phi) \sin (2 \pi m h / N)
$$

The error after symmetry averaging then becomes

$$
\begin{aligned}
\bar{e}_{m}(\theta) & =\frac{\sin (m \theta+\phi)}{N} \sum_{h=1}^{N} \cos (2 \pi m h / N) \\
& +\frac{\cos (m \theta+\phi)}{N} \sum_{h=1}^{N} \sin (2 \pi m h / N) .
\end{aligned}
$$

We next invoke trigonometric identities for the two summations. The identities in their standard form are

$$
\sum_{n=0}^{N} \sin (n x)=\frac{\sin (N x / 2) \sin ((N+1) x / 2)}{\sin (x / 2)}
$$

and

$$
\sum_{n=0}^{N} \cos (n x)=\frac{\cos (N x / 2) \sin ((N+1) x / 2)}{\sin (x / 2)} .
$$

Finally, we change the summation index from $n$ to $h$, evaluate the $h=0$ term explicitly to remove it from the summation, and insert $x=2 \pi m / N$. This produces the result that

$$
\sum_{h=1}^{N} \cos (2 \pi m h / N)=0
$$


and

$$
\sum_{h=1}^{N} \sin (2 \pi m h / N)=0,
$$

and therefore

$$
\bar{e}_{m}(\theta)=0
$$

when $m$ is not an integer multiple of $N$. This concludes the proof.

\section{Self-Correction}

At the level of approximation of interest here, on the order of 2 to $3 \mu \mathrm{rad}$, all encoder errors are in some sense associated with the disk. The largest error component in most cases is mode 1 , and it will be shown that this is due to imperfect disk centering. The next largest error component is typically mode 2, which is caused by disk tilt plus mode 2 error in the diffraction grating. Error modes 3 and higher are due entirely to errors in the disk itself. This includes errors in the grating lines and deviations from perfect flatness of the disk surface.

A key consideration is that disk centering error is [a] large and [b] varies with angle of rotation. Imperfections in the bearings such as small deviations from perfect sphericity cause the centering error to vary in a complicated way as a function of shaft rotation angle. Mechanical shock and thermal expansion also affect centering. Consequently, the mode 1 error component varies significantly with time and with shaft rotation angle. As for the magnitudes involved, in a 2.54 $\mathrm{cm}$ optical radius encoder, $1 \mu \mathrm{m}$ of centering error causes a maximum angular error of $1 \mu \mathrm{m} / 2.54 \mathrm{~cm} \doteq 40 \mu \mathrm{rad} \doteq 8$ arcsec, an order of magnitude greater than the level of accuracy sought here.

Because it is both large and time-dependent, disk centering error would seem to pose a major obstacle to high accuracy. For example, the traditional method of encoder mapping is of little use, because the correction map is static. Fortunately, this is precisely where the encoder theorem comes into play: if the encoder has two or more equally spaced read heads whose measurements are averaged together, then mode 1 error cancels out.

The next largest source of error in most cases is disk tilt. Simulations using the kinematic model described below show that disk tilt produces an error profile that is primarily mode 2. Like disk centering, disk tilt is a complicated function of time and rotation angle because of small imperfections in the bearings and races, mechanical shock and temperature variations. But this error can also be removed with the help of the theorem, this time by averaging together three or more read heads.

All that is required to satisfy the assumptions underlying the theorem is that the error at any particular instant of time must be describable by a Fourier series. Thus the encoder theorem provides real time self correction capability for some error modes, including the largest and most unstable.

This is all very encouraging, but there are limitations. There is a hardware cost associated with each additional read head, and there is limit on the number of read heads which will physically fit. Also the read heads are never perfectly placed in practice. Simulations show that read head placement errors lead to modal leakage: modes which ideally would cancel out identically do not in actuality disappear completely. Finally there remain the so-called degenerate modes, i.e. modes $N$, $2 N, 3 N, \ldots$, which are not corrected at all.

\section{SElf-COMPENSATION}

In some applications, the encoder theorem alone is not enough to reach the desired accuracy. An additional, complementary method is needed. One standard method is the reference mapping. A traceable reference encoder is attached to the axis of the encoder under test, and the axis is moved one full revolution in small steps. After each step, the rotation angle is measured by both encoders. The differences between the two encoder readings are computed and saved in an array called an encoder map which is then used to correct subsequent measurements.

The reference mapping method is in common use but is not well suited for high volume production. A trained operator must mount and dismount the reference encoder and monitor the test. Special adaptor hardware is required to mount the reference encoder, and the hardware is subject to damage and wear, causing accuracy to degrade over time. Accuracy also suffers if torque specifications are not rigidly adhered to when bolting the reference encoder on. The reference encoder and associated electronics and software are costly. Even when the adaptor hardware is in good condition and procedures are properly followed, the act of mounting the reference encoder physically disturbs the encoder under test, introducing repeatability errors on the order of an arc second. Finally, the reference encoder compensation can only be performed in a specially equipped facility. If an encoder drifts in the field, it must be sent back to the factory to be re-compensated, leading to significant down time for the user.

A better method is self-compensation, which requires no reference encoder, no physical contact, and no human operator. All that is needed is a single additional read head, called the reference head, which is located asymmetrically relative to the other read heads.

The method rests on the same assumptions as the encoder theorem and involves pairing each read head with the reference head. Then, for each pair, one computes the difference in the readings at a number of different angles. Consider one such pair; the first member is read head 0, i.e. the reference head, and the second is read head $n$. The error associated with read head 0 can be approximated by a Fourier series:

$$
e_{0}(\theta) \equiv e(\theta)=\sum_{j=1}^{M} S_{j} \sin j \theta+C_{j} \cos j \theta
$$

where $j$ is the mode number and $M$ is the mode at which the series is truncated. $e(\theta)$ is the error function of the encoder. It is in general a good approximation because the amplitude of the individual modes drops off rapidly with increasing mode 
number for a properly constructed encoder. The error for read head $n$ differs from that of read head 0 by a phase:

$$
e_{n}(\theta)=\sum_{j=1}^{M} S_{j} \sin \left(j \theta+j h_{n}\right)+C_{j} \cos \left(j \theta+j h_{n}\right)
$$

where $\theta$ is the shaft rotation angle and $h_{n}$ is the angular position of read head $n$ relative to read head 0 .

The error coefficients $S_{j}$ and $C_{j}$ must be somehow determined in order to correct subsequent encoder measurements. Ideally one would extract the coefficients by calculating the discrete Fourier transform of $e_{n}(\theta)$, but the reference encoder has been eliminated and so $e_{n}(\theta)$ cannot be measured directly. What can be measured instead are the read head differences, defined as

$$
d_{n}(\theta) \equiv e_{n}(\theta)-e_{0}(\theta)
$$

and then describe them by a different but related Fourier series

$$
d_{n}(\theta)=\sum_{j=1}^{M} S_{j}^{\prime} \sin \left(j \theta+j h_{n}\right)+C_{j}^{\prime} \cos \left(j \theta+j h_{n}\right)
$$

In this approach, the read head differences $d_{n}(\theta)$ are directly measured and a discrete Fourier transform or least squares Fourier fit is used to determine the $S^{\prime}$ and $C^{\prime}$ coefficients.

It will be shown (see section IV for a derivation) that the desired error coefficients $S_{j}$ and $C_{j}$ can be computed from the known difference coefficients $S_{j}^{\prime}$ and $C_{j}^{\prime}$ by a pair of simple equations. These equations, called the cotangent equations, are

$$
\begin{aligned}
S_{n j} & =\frac{1}{2}\left(C_{j}^{\prime} \cot \left(\frac{j h_{n}}{2}\right)-S_{j}^{\prime}\right) \\
C_{n j} & =-\frac{1}{2}\left(S_{j}^{\prime} \cot \left(\frac{j h_{n}}{2}\right)+C_{j}^{\prime}\right) .
\end{aligned}
$$

\section{A. Determination of Error Function Coefficients}

The following steps are used to determine the desired Fourier error coefficients from measurements from a pair of encoder read heads. Read head 0 is defined as the reference head and is by definition located at angle zero. The second read head is number $n$ and is located at angle $h_{n}$ relative to the reference head.

1) Specify $M$, the highest error mode to be corrected.

2) Specify $T$, the number of steps per full revolution.

3) Step the mechanical axis from 0 to $2 \pi$ radians in equal increments.

4) After each step, measure the rotation angle with read head 0 (the reference head), and read head $n$.

5) Compute the differences of the readings and store them in an array.

6) Compute the FFT of the differences array.

7) Extract the lowest $M$ difference DFT coefficients from the DFT.

8) Compute the lowest $M$ Fourier error coefficients with equations 16
The end result is that the coefficients $S_{j}$ and $C_{j}$ in equations 12 and 13 are determined out to the maximum desired mode $M$. In other words, a truncated Fourier series approximation of encoder error function $e(\theta)$ has been obtained.

\section{B. Multiple Read Heads}

The procedure requires the existence of at least two read heads, one of which is the reference head. In most cases there will be more than two read heads. Consider for example a socalled 2+1 design which has two equally spaced read heads with a spacing of 180 degrees plus a reference head which is located asymmetrically. In this case the reference head can be paired with two different read heads and the above procedure yields two separate sets of $S_{j}$ and $C_{j}$ coefficients.

Ideally both sets of coefficients are identical, but in reality the two sets of coefficients will differ slightly. It is therefore advantageous to average the two sets of coefficients together to obtain a single set which is more accurate than either of the individual sets. It is also useful to compute the deviations from the average as this provides useful diagnostic data regarding the health of the encoder hardware. They are also useful in choosing the truncation point $M$ of the Fourier series.

\section{Computing the encoder map}

The final step in the encoder self compensation process is extraction of the encoder map function $m(\theta)$. Note that $m(\theta) \neq e(\theta)$, although the two functions are closely related. $m(\theta)$ is calculated with a modified version of the error function equation 13 . The encoder theorem removes certain modes and so these modes need to be excluded from the summation. For an encoder with $N$ equally spaced read heads the equation is

$$
m(\theta)=\sum_{j=1}^{M / N} S_{N j} \sin N j \theta+C_{N j} \cos N j \theta
$$

As an example, consider a 3+1 type encoder with maximum mode number $M=9$. The summation is then

$$
\begin{aligned}
m(\theta) & =S_{3} \sin 3 \theta+C_{3} \cos 3 \theta \\
& +S_{6} \sin 6 \theta+C_{6} \cos 6 \theta \\
& +S_{9} \sin 9 \theta+C_{9} \cos 9 \theta
\end{aligned}
$$

Finally, the desired encoder map is just an array of values which comes from sampling the residual error function at a series of equally spaced discrete points. In other words, the final map is just

$$
m_{i} \equiv m\left(\theta_{i}\right), i=1,2,3, \ldots, i_{\max }
$$

The dimensionality of the map, $i_{\max }$, should be chosen in such a way that the highest mode of interest is sampled well.

\section{Positioning the reference head}

The position of the reference head must be chosen with care; a poor choice causes the cotangent values in eq. 16 to blow up, making the map hypersensitive to small errors in the raw measurements. In fact it is desirable to minimize the cotangents. This still leaves the designer with plenty of leeway. 


\section{Derivation of Cotangent Equations}

In this section we derive the cotangent equations 16 which are the basis of encoder self-compensation. We will model the individual read head errors by a Fourier series, and we will assume that the errors are the same for all read heads except for a phase angle. The measurement $\mu_{0}(\theta)$ from the reference head can be expressed in the following way:

$$
\mu_{0}(\theta)=\theta+\sum_{j=1}^{M} S_{j} \sin (j \theta)+C_{j} \cos (j \theta)
$$

where $j$ is the Fourier mode number, $\theta$ is the true shaft rotation angle and the summation represents the error. For read head $\mathrm{n}$, which is located at angle $h_{n}$ relative to the reference head, we get a similar expression but with phase terms added:

$$
\mu_{n}(\theta)=\theta+\sum_{j=1}^{M} S_{j} \sin \left(j \theta+j h_{n}\right)+C_{j} \cos \left(j \theta+j h_{n}\right)
$$

Now take the difference of the two measurements, which gives

$$
\begin{aligned}
d_{n}(\theta)= & \mu_{n}(\theta)-\mu_{0}(\theta) \\
= & \sum_{j=1}^{M} S_{j} \sin \left(j \theta+j h_{n}\right)+C_{j} \cos \left(j \theta+j h_{n}\right) \\
& \quad-S_{j} \sin (j \theta)-C_{j} \cos (j \theta)
\end{aligned}
$$

Note that when the difference of two read heads is taken, the true rotation cancels out and the remaining expression is due solely to the errors in the two readings. We now want to cast the expression for the read head differences into a more useful form. To begin with, we use trigonometric identities [12] to rewrite the summand as

$$
\begin{aligned}
& 2 \sin \left(\frac{j h_{n}}{2}\right) \\
& \cdot\left[S_{j} \cos \left(j \theta+\frac{j h_{n}}{2}\right)-C_{j} \sin \left(j \theta+\frac{j h_{n}}{2}\right)\right]
\end{aligned}
$$

Then we make the substitutions

$$
\begin{aligned}
S_{j}^{\prime \prime} & \equiv 2 S_{j} \sin \left(\frac{j h_{n}}{2}\right) \\
C_{j}^{\prime \prime} & \equiv-2 C_{j} \sin \left(\frac{j h_{n}}{2}\right)
\end{aligned}
$$

which yields

$$
d_{n}(\theta)=\sum_{j=1}^{M} S_{j}^{\prime \prime} \cos \left(j \theta+\frac{j h_{n}}{2}\right)+C_{j}^{\prime \prime} \sin \left(j \theta+\frac{j h_{n}}{2}\right)
$$

For our final simplification step we define new coefficients $S_{j}^{\prime}$ and $C_{j}^{\prime}$ which satisfy the equation

$$
\begin{aligned}
& S_{j}^{\prime \prime} \cos \left(j \theta+\frac{j h_{n}}{2}\right)+C_{j}^{\prime \prime} \sin \left(j \theta+\frac{j h_{n}}{2}\right) \\
& \equiv C_{j}^{\prime} \cos j \theta+S_{j}^{\prime} \sin j \theta
\end{aligned}
$$

so that

$$
d_{n}(\theta)=\sum_{j=1}^{M} C_{j}^{\prime} \cos j \theta+S_{j}^{\prime} \sin j \theta
$$

To obtain explicit expressions for the new coefficients, first set $\theta=0$ in the above equation. This immediately gives

$$
C_{j}^{\prime}=S_{j}^{\prime \prime} \cos \left(\frac{j h_{n}}{2}\right)+C_{j}^{\prime \prime} \sin \left(\frac{j h_{n}}{2}\right)
$$

Next, setting $\theta=\pi / 2$ gives

$$
S_{j}^{\prime}=S_{j}^{\prime \prime} \cos \left(\pi / 2+\frac{j h_{n}}{2}\right)+C_{j}^{\prime \prime} \sin \left(\pi / 2+\frac{j h_{n}}{2}\right)
$$

or equivalently

$$
S_{j}^{\prime}=-S_{j}^{\prime \prime} \sin \left(\frac{j h_{n}}{2}\right)+C_{j}^{\prime \prime} \cos \left(\frac{j h_{n}}{2}\right)
$$

Now solve for $S_{j}^{\prime \prime}$ and $C_{j}^{\prime \prime}$ :

$$
\begin{aligned}
& S_{j}^{\prime \prime}=C_{j}^{\prime} \cos \left(\frac{j h_{n}}{2}\right)-S_{j}^{\prime} \sin \left(\frac{j h_{n}}{2}\right) \\
& C_{j}^{\prime \prime}=S_{j}^{\prime} \cos \left(\frac{j h_{n}}{2}\right)+C_{j}^{\prime} \sin \left(\frac{j h_{n}}{2}\right)
\end{aligned}
$$

Substituting the definitions of $S_{j}^{\prime \prime}$ and $C_{j}^{\prime \prime}$ into these equations gives

$$
\begin{aligned}
& 2 S_{j} \sin \left(\frac{j h_{n}}{2}\right)=C_{j}^{\prime} \cos \left(\frac{j h_{n}}{2}\right)-S_{j}^{\prime} \sin \left(\frac{j h_{n}}{2}\right) \\
& -2 C_{j} \sin \left(\frac{j h_{n}}{2}\right)=S_{j}^{\prime} \cos \left(\frac{j h_{n}}{2}\right)+C_{j}^{\prime} \sin \left(\frac{j h_{n}}{2}\right)
\end{aligned}
$$

which can then be rearranged to give the desired result:

$$
\begin{aligned}
S_{j} & =\frac{1}{2}\left(C_{j}^{\prime} \cot \left(\frac{j h_{n}}{2}\right)-S_{j}^{\prime}\right) \\
C_{j} & =-\frac{1}{2}\left(S_{j}^{\prime} \cot \left(\frac{j h_{n}}{2}\right)+C_{j}^{\prime}\right)
\end{aligned}
$$




\section{Simulations}

\section{A. Kinematic Model}

As a first step in the validation of the foregoing formalism, a series of simulations was run. These simulations are based on a simple kinematic model of angular encoders which will now be described.

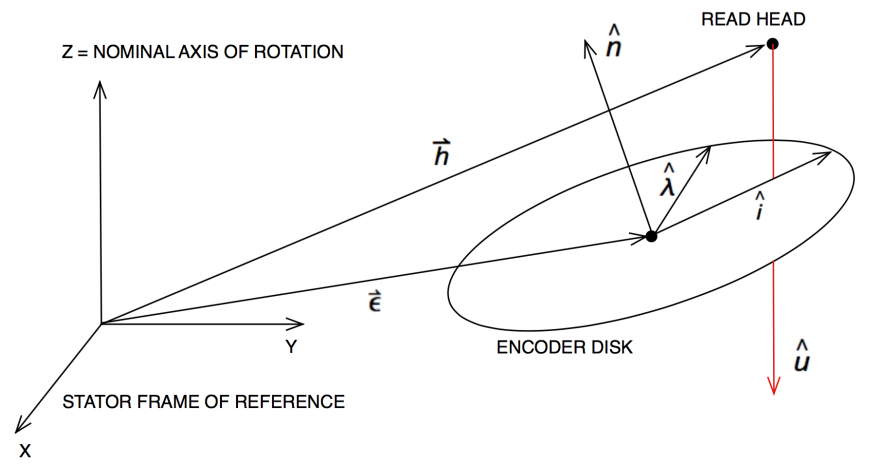

Fig. 4. Encoder kinematic model

Figure 4 illustrates the basic ideas behind the kinematic model. We begin by defining the stator frame of reference, in which the read head has a fixed position. The $Z$ axis is defined to coincide with the nominal axis of rotation. The $X$ and $Y$ axes are perpendicular to $\mathrm{Z}$ and obey the right hand rule and are oriented such that the $Y$ coordinate of the read head is zero. For clarity, all variables will be defined in the stator frame and all calculations are carried out in this frame.

The encoder disk is modeled by three vectors. The position vector of the disk center (more specifically the center of the radial diffraction grating that is etched on the disk surface) is $\vec{\epsilon}$, the normal vector of the disk is $\hat{n}$, and the unit vector which represents the index line on the disk grating is $\hat{\lambda}$. In the ideal case of a perfectly centered and un-tilted disk, $\vec{\epsilon}=0$ and $\hat{n}=(0,0,1)$. The index line vector $\hat{\lambda}$ defines the zero point for angle measurements on the disk.

The read head is modeled as a fixed point in space with position vector $\vec{h}$, which emits a beam with direction vector $\hat{u}$. The beam intersects the plane of the disk at a point whose direction vector relative to the disk center is $\hat{i}$. What the read head measures, in effect, is the angle between the intersection vector $\hat{i}$ and the index line vector $\hat{\lambda}$.

The steps for computing the expected measurement from a single read head for a given angular encoder configuration can now be listed:

1) Specify encoder configuration parameters:

$\begin{array}{ll}\hat{a} & \text { Shaft rotation axis } \\ \vec{h} & \text { Read head position vector } \\ \hat{u} & \text { Read head beam direction vector } \\ \vec{\epsilon}_{0} & \text { Disk position vector before shaft rotation } \\ \hat{n}_{0} & \text { Disk normal vector before shaft rotation } \\ \hat{\lambda}_{0} & \text { Initial disk index line direction vector }\end{array}$

2) Specify shaft rotation angle $\theta$
3) Compute rotation matrix $M(\theta, \hat{a})$

4) Rotate disk configuration vectors:

$$
\begin{aligned}
& \vec{\epsilon}=M \cdot \vec{\epsilon}_{0}, \\
& \hat{n}=M \cdot \hat{n}_{0}, \\
& \hat{\lambda}=M \cdot \hat{\lambda}_{0}
\end{aligned}
$$

5) Compute intersection $\vec{i}$ of read head beam with plane of rotated disk

6) Compute intersection direction vector $\hat{i}=N(\vec{i}-\vec{\epsilon})$, where $N()$ indicates normalization

7) Compute angle $\theta^{\prime}$ between disk intersection direction vector $\hat{i}$ and rotated index line direction vector $\vec{\lambda}$.

8) Compute error in read head measurement $e=\theta^{\prime}-\theta$

The intersection $\vec{i}$ of a line described by a point $\vec{r}$ and direction vector $\hat{u}$ with a plane described by a point $\vec{p}$ and normal vector $\hat{n}$ is

$$
\vec{i}=\frac{\vec{p} \cdot \hat{n}-\vec{r} \cdot \hat{n}}{\hat{u} \cdot \hat{n}}
$$

Next we give the computational steps to determine the angle $\theta$ between two unit vectors $\hat{a}$ and $\hat{b}$ relative to a known normal vector $\hat{n}$ that produces a result on the interval $[-\pi, \pi]$ :
1) $d=\hat{a} \cdot \hat{b}$
2) $\vec{c}=\hat{a} \times \hat{b}$
3) $c=|\vec{c}|$
4) $s=S(\vec{c} \cdot \hat{n})$
5) $\theta=\arctan (s c, d)$

where the function $S()$ returns the sign of the argument.

This model can be adapted to simulated any number of read heads. It is also possible to model errors in the disk itself, i.e. grating errors, with any desired functional form.

\section{B. Simulation Results}

With the kinematic model, simulations can be constructed which illustrate the effects of disk centering and disk tilt errors. It will be recalled that these errors vary with time and shaft rotation angle because of imperfections in the bearings, including slight variations in radius from one ball to another and slight deviations from sphericity of each individual ball. A static additive correction is therefore not effective for removing these errors, and real time self-correction based on symmetry cancellation and the encoder theorem must be used instead.

Figure 5 shows that disk centering error produces a large mode 1 Fourier component, with an admixture of higher modes which are too small to be visible on the plot. Similarly, figure 6 shows that disk tilt produces a mode 2 component, again with invisibly small amounts of higher modes.

Figure 7 illustrates the encoder theorem in action for the case of an encoder with an optical radius of $2.54 \mathrm{~cm}$ with $5 \mu \mathrm{m}$ centering error, $6 \mathrm{mrad}$ disk tilt error, $5 \mu$ rad mode 3 grating error and $2.5 \mu$ rad mode 4 grating error. Error modes 1 though 4 are present in this case, and the residual error plot 
shows that only the $5 \mu$ rad mode 3 signal survives symmetry averaging; this is in keeping with the prediction of the theorem.

The final simulation plot, figure 8, is an example of the encoder theorem and self-compensation operating in tandem for the case of an encoder with an optical radius of 2.54 $\mathrm{cm}$ with $5 \mu \mathrm{m}$ centering error, $6 \mathrm{mrad}$ disk tilt error, $5 \mu \mathrm{rad}$ mode 3 grating error and $2.5 \mu \mathrm{rad}$ mode 4 grating error. In this case, errors modes 1 through 4 are initially present. Symmetry cancellation effectively removes modes 1,2 and 4 while selfcompensation addresses mode 3 . As shown in the plot, the remaining error is mode 6 as expected, with an amplitude that is a tiny fraction of a $\mu \mathrm{rad}$.

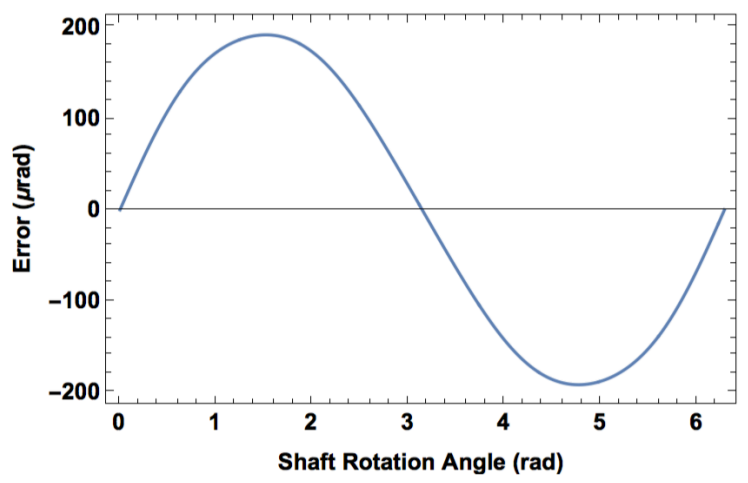

Fig. 5. Error function for $r=2.54 \mathrm{~cm}$ encoder, $5 \mu \mathrm{m}$ centering error

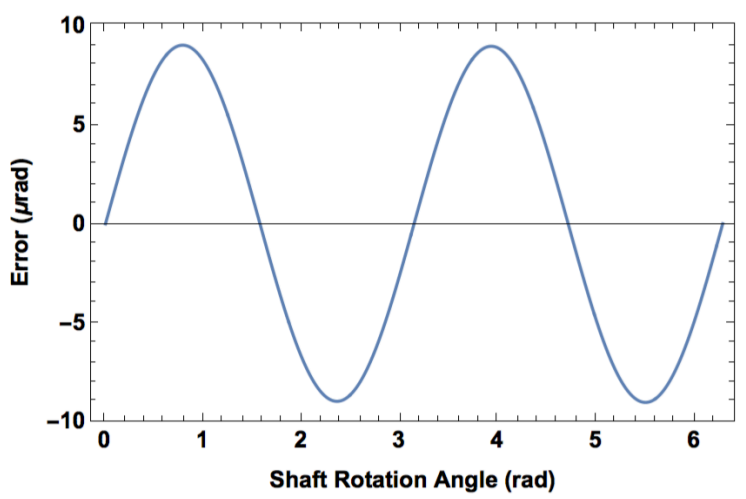

Fig. 6. Error function for $r=2.54 \mathrm{~cm}$ encoder, $6 \mathrm{mrad}$ disk tilt error

\section{Limitations and Error Sources}

At this point some cautionary notes are in order. The intention of the simulations presented above was to [a] validate the formalism and $[b]$ to illustrate the formalism in action. The extremely small errors that remained in these simulations after correction and self compensation are not achievable in reality. For one thing, in a realistic scenario the read heads are not perfectly placed, and imperfect placement leads to modal leakage: error modes that would ideally be completely removed in fact remain in small residual amounts.

There is also a limitation on the number of modes which can be addressed. These methods are good for low angular

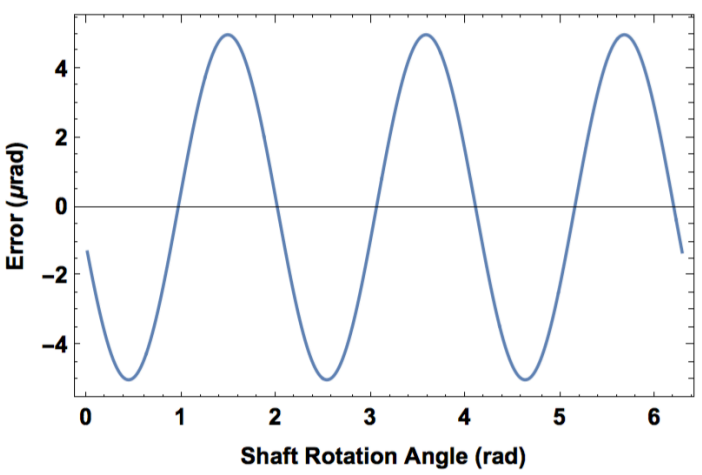

Fig. 7. Error function for $r=2.54 \mathrm{~cm}$ encoder with $5 \mu \mathrm{m}$ centering error, 6 mrad disk tilt error, $5 \mu \mathrm{rad}$ mode 3 grating error and $2.5 \mu \mathrm{rad}$ mode 4 grating error, after three head symmetry cancellation

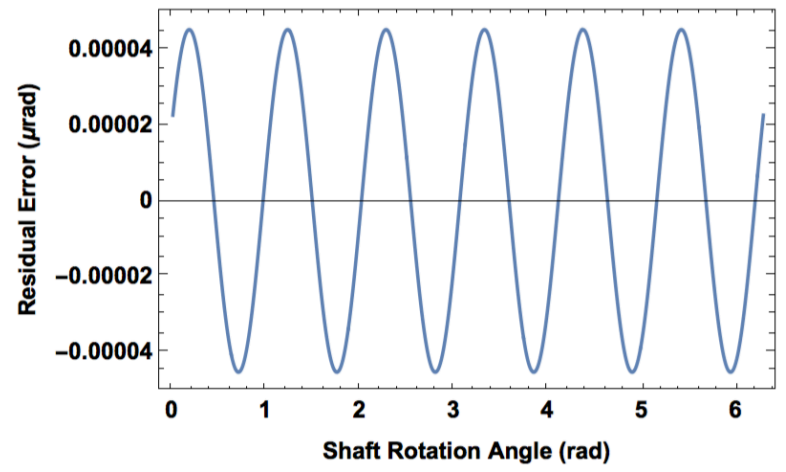

Fig. 8. Error function for $r=2.54 \mathrm{~cm}$ encoder with $5 \mu \mathrm{m}$ centering error, 6 mrad disk tilt error, $5 \mu \mathrm{rad}$ mode 3 grating error and $2.5 \mu \mathrm{rad}$ mode 4 grating error, after three head symmetry cancellation and mode 3 self compensation

frequency modes, but higher modes are problematic. For example, if the mode in question has a wavelength that is comparable to or smaller than the read head placement tolerance, then it will not be removed by these methods. This means that the methods presented here work best with encoder disks that do not have high frequency error modes. Fortunately this turns out to be true for the vast majority of cases.

As with other Fourier methods, phenomenon of aliasing needs to be considered. This issue can be mitigated by appropriate filtering during data collection.

There is also the question of what level of encoder accuracy is suitable for the application. The encoder is only as good as the enclosing structure. If the enclosing structure, typically a robot joint, is subject to other errors such as bending and thermal expansion, then the accuracy of the encoder may not produce the desired overall system accuracy. In other words, to achieve high accuracy for a robotic system, high accuracy encoders are necessary but not sufficient. What has been presented here can be a part of the answer but it is not the entire answer. 


\section{TEST RESUlts}

In addition to simulations, physical tests have been carried out in which encoder maps obtained by self-compensation were compared to maps obtained by reference-mapping. The reference encoder in these tests was a Heidenhain RON-901 and the encoder under test was a $3+1$ type: 3 equally spaced read heads plus one asymmetrically mounted reference head.

A sample test result is shown in figure 9. The plot compares a map function obtained by self-compensation with a map obtained from the RON-901. Recall that the map function is a measure of the error that remains after symmetry averaging. The plot indicates that averaging has left an error signal that is primarily mode 3 as expected from the encoder theorem. The amplitude of the map is approximately $15 \mu \mathrm{rad}$; for comparison, the amplitude before averaging was on the order of $150 \mu \mathrm{rad}$.

The maximum difference between the two maps is approximately $5 \mu \mathrm{rad}$ or 1 arcsecond. In fact, subsequent testing done by indirect non-contact methods showed that the maximum error after self-compensation was actually slightly less. The dominant error in this test is actually the attachment hardware.

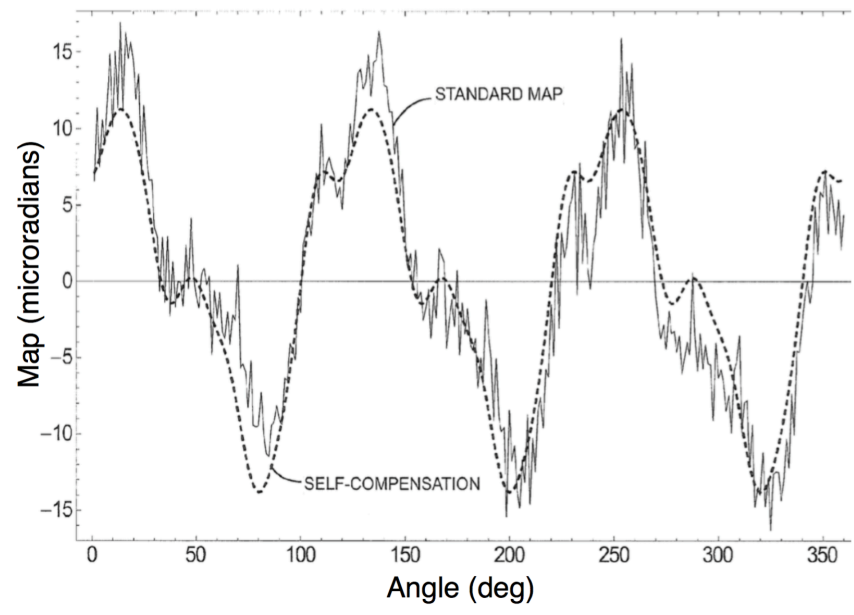

Fig. 9. Comparison of post symmetry averaging encoder maps obtained by self-compensation (dotted line) and reference mapping (solid line)

\section{A. Indirect Tests}

The $3+1$ encoder design discussed above has been standard equipment in popular commercial laser tracker devices for the dimensional metrology market for several years now. Customers include the largest and most demanding players in the aerospace and automotive industries. Before shipping, each of these devices is subjected to stringent NIST-traceable calibration procedures which follow the ASME B89.4.1 standard [13]. These are system-level tests which quantify the accuracy of the 3D coordinate measuring capability of each tracker. These tests provide excellent indirect measures of encoder accuracy. In addition, each tracker is subjected to so-called interim tests each time they are prepared for a measuring job. These are also stringent indirect tests of encoder accuracy.
Years of experience have shown that these systems function within specifications [15] indefinitely without human intervention.

\section{CONCLUSIONS}

Two complementary methods for improving the accuracy and stability of optical robotic angular encoders have been presented, and their consequences worked out. Both methods are based on Fourier analysis of encoder errors. In addition, a method for simulating realistic angular encoder operational scenarios was presented.

The encoder theorem involves averaging $N \geq 2$ uniformly spaced read heads, and it was proven that in this case all Fourier error modes cancel out identically except for degenerate mode $N$ and its harmonics, which are unaffected.

The encoder theorem is useful because it provides real time self-correction capability. It is especially useful for modes 1 and 2, which simulations showe are due to disk centering and disk tilt errors respectively. Because these modes are sensitive to the instantaneous state of the bearings, they are inherently volatile and cannot be accurately corrected with any static method such as an encoder map.

We then presented a self-compensation method which uses an asymmetrically placed reference read head to measure the degenerate modes and produce an error map which can then be used to correct subsequent measurements. Self-compensation is well suited for modes which are relatively stable over time such as errors in the disk grating. When used in tandem, these two methods enable the construction of stable, cost-effective encoders with sub-arcsecond accuracy.

These methods are well suited for robotic applications because accuracy is maintained with zero human intervention. Self-correction occurs automatically with each reading the encoder makes, while self-compensation is easily automated and can be initiated automatically as well if desired. They also enable the encoder to continuously monitor its own health.

A kinematic model of angular encoders was then presented. This model was used to construct simulations which demonstrate that disk centering and disk tilt errors produce primarily mode 1 and mode 2 Fourier components respectively. They were also used to demonstrate the operation of the encoder theorem and self-compensation methods in action. The kinematic model is also useful for encoder design; different read head arrangements and different mechanical tolerances and different measurement scenarios can all be simulated.

Finally a sample test result was shown to illustrate the effectiveness of these methods in a real encoder. Taken together, the methods given here provide a practical approach for designing practical angular encoders with sub-arcsecond accuracy and which require no human intervention to maintain their accuracy over long periods of time. Source code and additional examples are available for download [14].

\section{ACKNOWLEDGEMENTS}

Author acknowledges the support of Spatial Metrix, Inc. and FARO Technolgies, Inc., and thanks Robert Bridges and 
John Hoffer for many useful discussions. Author also thanks Jonathan Day, Philip Dunwody, Jeremy Garey, Robert Landgrebe, John Manfield, Nils Steffensen, Christopher Thawley, James West and the entire Kennett Square team for their unflagging help and encouragement.

\section{REFERENCES}

[1] W.T. Estler, Y.H. Queen, J. Bryan, An Advanced Angle Metrology System, CIRP Annals - Manufacturing Technology, Volume 42, Issue 1, 1993, Pages 573-576

[2] Ralf D Geckeler, Alfred Link, Michael Krause and Clemens Elster, Capabilities and limitations of the selfcalibration of angle encoders, Measurement Science and Technology, Volume 25, Number 5,14 March 2014

[3] R Probst, Self-calibration of divided circles on the basis of a prime factor algorithm, Measurement Science and Technology, Volume 19, Number 1

[4] Tsukasa Watanabe, Hiroyuki Fujimoto and Tadashi Masuda, Self-Calibratable Rotary Encoder, Journal of Physics: Conference Series 13 (2005)

[5] Masuda, T., and M. Kajitani, High accuracy calibration system for angular encoders, Journal of Robotics and Mechatronics 5.5 (1993): 448-452

[6] Tsukasa Watanabe, Is an angular standard necessary for rotary encoders?, Synthesiology, Vol.1, No.4, p.296-304 (2008)

[7] Masuda T and Kajitani M, An automatic calibration system for angular encoders, Precision Engineering, Volume 11, Issue 2, 1989, Pages 95-100

[8] Just, A., et al., Comparison of angle standards with the aid of a high-resolution angle encoder, Precision engineering 33.4 (2009): 530-533

[9] Lu, X-D., et al., On-axis self-calibration of angle encoders, CIRP Annals-Manufacturing Technology 59.1 (2010): 529-534

[10] Cramer, P. G., Self Compensating Angular Encoder, U.S. Patent 9,234,773, issued January 12, 2016.

[11] Wolfram MathWorld/Sine

[12] Wolfram MathWorld/Cosine

[13] Methods for Performance Evaluation of Coordinate Measuring Machines (ASME Standard B89.4.1 - 2006), Www.asme.org

[14] github.com/pgcramer/RSS

[15] Faro Laser Tracker brochure, www.faro.com

[16] Founder Cramer Consulting LLC, cramermath.com 